\title{
POLITIICAS INSTITUCIONALES Y TRABAJO COLABORATIVO ENTRE DOCENTES: EL EJEMPLO DE LA ZONA CLIC
}

\section{José Peirats Chacón, Cristina Sales Arasa (*)}

SÍNTESIS: Es incuestionable el proceso de inmersión tecnológica que hoy experimenta la institución escolar. Iniciativas públicas y privadas están interesadas en incorporar las tecnologías de la información ( $\mathrm{Tl}$ en lo sucesivo) al sistema escolar. Centrándonos en las primeras, y dentro del marco político de España, describimos brevemente algunos proyectos estatales de apoyo a las TI en las escuelas, tras lo cual nos fijamos en el caso del programa Clic, una aplicación educativa abierta y libre desarroIlada en la Comunidad Autónoma de Cataluña, caracterizada por el trabajo colaborativo de miles de docentes de diversas partes del mundo.

(*) Profesores de Educación Secundaria y asociados del Departamento de Didáctica y Organización Escolar, Universidad de Valencia, España. 


\section{INTRODUCCIÓN}

En este trabajo nos proponemos reflexionar a propósito del proceso de inmersión tecnológica de la institución escolar, empeño que afrontamos a partir de una serie de iniciativas de carácter público que consideramos significativas acerca de cómo las tecnologías de la información, sobre todo las de última generación ( TI), se incorporan al sistema escolar reglado. En esta ocasión no trataremos de las prácticas que se realizan en las aulas, sino de la compleja trama de proyectos y de programas diseñados para situar a la institución escolar en la llamada sociedad de la información y de la comunicación. Revisar la gran cantidad de iniciativas surgidas en este ámbito no sería posible aquí, razón por la cual prestaremos especial atención al Programa Clic, a la aparición, distribución y generalización de un programa educativo abiertoy libre como es éste, en la medida en que nos parece ejemplarizador de un modo de proceder en el sector.

La sociedad de la información descansa en los incesantes y cada vez mayores flujos de datos, de imágenes y de sonidos que recorren vertiginosamente el ciberespacio, lo cual es posible gracias a la disposición de redes y de artefactos de TI. En estos momentos el nivel de penetración de estas tecnologías en nuestras instituciones educativas va aumentando con rapidez, y se presentan como instrumentos mediadores en el proceso de transmisión de los conocimientos. En este proceso, y como parte de las investigaciones dirigidas a la realización de nuestras respectivas tesis ${ }^{1}$, nos planteamos cuestiones tales como: ¿qué papel le corresponde jugar a la escuela en la nueva sociedad de la información? ¿Es posible la colaboración entre el profesorado para crear un nuevo entorno tecnológico? ¿Qué condiciones son favorables para la aplicación de las TI por los docentes en su quehacer cotidiano?

\section{EL MARCO POLÍTICO DE LAS TECNOLOGÍAS DE LA INFORMACIÓN}

La realidad del objeto de nuestra atención se encuentra en España, pero es necesario exponer, aunque sea de forma sucinta, al gunas

1J Peirats Chacón: «Variantes organizativas generadas por las tecnologias de la información. Un estudio en los centros de Primaria de la Comunidad Valenciana». C. Sales Arasa: «Análisis de las estrategias de enseñanza con tecnologías de la información. ¿Un nuevo contexto metodológico en Secundaria?». 
ideas sobre su estructura territorial para comprender con nitidez los aspectos más relevantes de la implantación de algunos de los planes institucionales de integración de los medios tecnológicos en los centros educativos.

- El Estado español forma parte de una alianza política, como es la Unión Europea, con una Constitución que en estos momentos se encuentra pendiente de aprobación por los distintos países que la integran, y en la que las directrices que emanan de sus órganos de poder deben cumplirse en los ámbitos nacionales. Algunas de ellas afectan al sistema escolar, y, por supuesto, al compromiso europeo con la expansión de la sociedad de la información. Así, el Consejo de Europa, en la Cumbre de Lisboa, percibe la iniciativa eLearning como concebir la educación del futuro a través de cuatro líneas de actuación: infraestructuras y equipamiento, formación, contenidos y servicios europeos. Estos planteamientos sellevan a cabo mediante programas específicos en cada Estado miembro. En España se puso en marcha elPlan Info XXI, y, tras su evaluación, a finales de 2003, se sustituyó por el plan España.es, recientemente vuelto a cambiar por el de Todos.es ${ }^{2}$. Con independencia de algunos matices, la idea maestra de todas estas iniciativas políticas es la misma: ayudar con financiación y con formación a que los ciudadanos se sumerjan en la sociedad de la información por medio de acciones de fomento de las TI en las administraciones central, autonómica y local, en el mundo laboral, y en los servicios o en las escuelas y universidades.

- Por otro lado, la Constitución española permite que parte de las competencias políticas, legislativas y ejecutivas estatales sean ejercidas por los órganos democráticos que gobiernan en las distintas comunidades autónomas que integran el Estado. En la actualidad todas ellas disfrutan de competencias en materia de educación, y, por tanto, disponen de la capacidad de adoptar iniciativas y proyectos de

\footnotetext{
${ }^{2}$ En las elecciones generales de marzo de 2004 el Partido Socialista (PSOE) consiguió mayoría suficiente para acceder al Gobierno, y la polémica suscitada por los anteriores planes invitó a formular un plan alternativo a los gestionados por el Partido Popular. De cualquier modo, las acciones concretas de Todos.es no se conocerán hasta finales de 2005.
} 
equipamiento tecnológico en los centros escolares bajo su jurisdicción; no obstante, cuentan con órganos (la Conferencia de Consejeros de Educación y el Centro Nacional de Información y Comunicación Educativa) que tienen funciones de intercambio de información y de coordinación de las diversas políticas que las integran, aunque en la práctica estas reuniones resultan campos para la confrontación entre los representantes del gobierno central y los autonómicos, y entre los partidos que sustentan al gobierno de turno y a los de la oposición. Es por ello que se advierte una escasa coordinación entre los programas de las comunidades autónomas y los diseñados desde el ámbito estatal.

\section{INICIATIVAS ESTATALES DE APOYO A LAS TI EN LOS CENTROS ESCOLARES}

El Programa de Nuevas Tecnologías de la Información y la Comunicación (PNTIC), pionero en España, fue impulsado en 1987 por el Ministerio de Educación, Cultura y Deporte para fusionar los proyectos Atenea (sobre informática) y Mercurio (sobre medios audiovisuales, en especial el vídeo). Su objetivo fundamental consistía en la integración de las nuevas tecnologías en toda la red de centros de educación infantil, primaria y secundaria del Estado. Las iniciativas principales del PNTIC se canalizaron en cuatro áreas: la dotación de infraestructuras, el desarrollo de contenidos interactivos, la formación del profesorado, y el estudio del papel del centro y del profesorado.

Hoy el PNTIC ha sido sustituido por el Centro Nacional de Información y Comunicación Educativa ( CNICE) ${ }^{3}$, un ente administrativo dependiente del Ministerio, con funciones de animación y de coordinación de todas aquellas iniciativas relacionadas con el macroproyectol nfo XXI y con su proyección a los ámbitos escolares. En abril de 2002 estas iniciativas se plasmaron en el denominado Programa Internet en la Escuela, por el que se intenta dotar a los centros de conexiones con Internet de banda ancha, desarrollar software educativo multimedia, elaborar contenidos para la enseñanza obligatoria, formar al profesorado, adaptar los currículos para potenciar la utilización de las nuevas tecno-

\footnotetext{
${ }^{3}$ http://www.cnice.mecd.es.
} 
logías, e instruir a los padres de los alumnos y a otros miembros de la comunidad educativa en el uso de las TI.

Entre los proyectos que han surgido del PNTIC o del actual CNICE citamos los siguientes: el Proyecto Mentor, que atiende las necesidades de formación de las personas adultas; en la actualidad este proyecto está en fase de expansión, colaborando en él nuevas comunidades autónomas y algunos países de Latinoamérica. Otra iniciativa es la llamada Aldea Digital, que tiene la pretensión de integrar las TI en las escuelas rurales. Las Redes en Educación, cuyo objetivo principal es fomentar las Intranets y conectar entre sí los ordenadores (computadores) de los que dispone cada centro educativo. Las Aulas hospitalarias, que desde 1996 pretenden integrar las TI en la educación dirigida a la población hospitalizada en aras de superar el aislamiento del alumno que temporal o permanentemente está hospitalizado. EI Proyecto ABIES (aplicación para la gestión de bibliotecas escolares), que está centrado en la utilización de la informática como soporte en las bibliotecas escolares. Y, para finalizar en esta breve exposición de los planes que se están desarrollando, encontramos el Centro Virtual de Educación, que ofrece formación en línea para el alumnado de los distintos niveles de enseñanza reglada, para los profesores y para los adultos en general.

\section{TECNOLOGÍAS DE LA INFORMACIÓN EN LAS COMUNIDADES AUTÓNOMAS}

Describir aunque sea de forma sucinta las políticas sobre tecnologías de la información de las 17 comunidades autónomas que forman el Estado español, excede las pretensiones de este artículo, por lo que nos centraremos sólo en una: Cataluña. En concreto, sobre uno de los programas educativos estrella que viene desarrollando desde hace varios años: el Programa Clic. Ahora bien, para entender por qué este proyecto surge en Cataluña, es preciso incluir una breve pincelada contextual. Distintos informes reconocen que la Comunidad Autónoma de Cataluña es, dentro del Estado español, la que más pronto y con mayor intensidad se está incorporando a la sociedad de la información. Avalan esta apreciación datos como los siguientes: en 2003 , el $54,39 \%$ de los hogares catalanes disponía de uno o más ordenadores, y, de éstos, el $37,22 \%$ estaba conectado a Internet (ambos indicadores son muy superiores a la media del país). 
En el lado opuesto, y a modo de ejemplo, estaría la Comunidad Autónoma de Extremadura, en la que el $32,33 \%$ de los hogares tiene ordenador, y de aquéllos sólo está conectado a Internet el 14,77\%. Respecto a otro indicador, y pese a que en Cataluña la práctica totalidad de los centros públicos de primaria y de secundaria está conectado a Internet, sólo el $12 \%$ de ellos dispone de una web activa con diversos servicios a la comunidad escolar, mientras que en el caso de los centros públicos de Extremadura, con un porcentaje algo inferior de conexión a Internet, el 25,1\% tiene activa su página $w^{4}{ }^{4}$. El mejor equipamiento tecnológico de Cataluña representaría una de las condiciones necesarias, aunque no lo suficiente, para que se pueda desarrollar un proyecto de la naturaleza que aquí nos ocupa.

La Generalidad de Cataluña (que es como se denomina el gobierno de dicha Comunidad Autónoma), tiene plenas competencias en materia educativa desde principios de los años ochenta. Desde su Departamento de Educación se impulsó en 1986 el Programa de Informática para la Enseñanza (PIE), con objeto de coordinar las actuaciones encaminadas hacia la introducción de las nuevas tecnologías de la información en la enseñanza no universitaria. Un aspecto esencial de dicho Programa se refiere a Internet, y se concreta en la Red Telemática Educativa de Cataluña (XTEC) ${ }^{5}$, creada en 1988 y conectada a la red desde 1995. Más tarde se puso en marcha un proceso de remodelación de todas estas ofertas para hacerlas accesibles desde otro portal ${ }^{6}$, que ofrece tanto los servicios administrativos de la Generalidad como los pedagógicos concretos. Este nuevo diseño se ha hecho necesario, sobre todo a partir del creciente acceso a la información y a los servicios relativos a la educación de padres y de alumnos desde puntos ajenos a la red de la Generalidad. Algunos de los servicios que se ofrecen son:

Acceso para profesores y para centros docentes, que tiene tres objetivos: la formación permanente, la actividad académica con alumnos y la investigación educativa. Los centros educativos cuentan con un identificador institucional de correo y de web a disposición de la dirección del centro, y, además, se utiliza Internet para temas de tipo administrativo-educativo como las inscripciones en los cursos de forma-

\footnotetext{
${ }^{4}$ Informe de evaluación sobre el desarrollo de la Sociedad de la Información en España. eEspaña 2004. Madrid, Fundación Auna, 2004. (pp. 136 y ss.).

${ }^{5}$ http://www.xtec.es.

${ }^{6}$ http://www.edu365.com.
} 
ción. En estos momentos la práctica totalidad de los centros públicos, aparte de los privados, está conectado a la red digital de servicios integrados, y más de 68.000 docentes poseen cuenta de correo e identificador de acceso a la web.

Web de la propia XTEC y webs de centros. Contiene miles de recursos informativos y de documentación, y también puede alojar sus páginas web, si lo desea y sin costo alguno, cualquier centro público 0 privado de la Comunidad catalana.

Correo electrónico de los alumnos. Este servicio se proporciona mediante una normativa específica. Consiste en poner a disposición de las direcciones de los centros escolares un cierto número de cuentas de correo, que las asignan a los al umnos bajo su criterio, responsabilidad y control. Si para participar en un proyecto escolar se necesita que los 30 alumnos de un aula dispongan de correo electrónico, se les suministra hasta su finalización.

Video-on-demand. Permite hacer llegar a los centros, a través de la red, aquellos documentos en soporte vídeo que se encuentren digitalizados.

Entre otros proyectos en los que están inmersos, debemos destacar, en primer lugar, un concurso anual de webs de ciencia. Se invita a todos los centros a participar, aportando sus webs de tipo científico realizadas por los alumnos bajo la dirección de sus profesores. Se publica en Internet para que sea visible por toda la comunidad escolar. Otra línea de trabajo consiste en poner en Internet las tradicionales revistas escolares. También se potencian los proyectos colaborativos en campos como las lenguas extranjeras y la música, entre otras áreas del currículo.

\subsection{EL PROGRAMA CLIC, EJEMPLO DE ENTORNO ABIERTO A LA COLABORACIÓN DEL PROFESORADO}

El Clic es un programa informático educativo de difusión gratuita entre los docentes, creado a principios de la década de los noventa por Francesc Busquets ${ }^{7}$, un profesor de educación básica con

\footnotetext{
${ }^{7}$ http://www.xtec.es/ fbusquet
} 
experiencia en el uso de los ordenadores (computadoras) en la escuela, que fue invitado a formar parte del equipo técnico del PIE, en donde pudo desarrollarlo y ampliarlo. La versión en castellano fue editada por el PNTIC del Ministerio de Educación y Ciencia, gracias al premio obtenido en la convocatoria de Programas Educativos para Ordenador de 1992. La versión en catalán fue incluida en las distintas ediciones del CD-ROM «Sinera» del PIE y en la web de la XTEC. El uso del programa se fue extendiendo entre los seminarios de informática educativa y en los grupos de renovación pedagógica, que aportaron muchas ideas y sugerencias para ampliarlo y mejorarlo. La versión actual, la Clic 3.0, se encuentra disponible en siete idiomas: catalán, castellano, gallego, vasco, francés, inglés y alemán. En estos tres últimos idiomas se ha podido realizar gracias a un proyecto impulsado por la Comunidad Europeå.

EI Clic es un entorno (software) abierto y orientado sobre todo al profesorado. Con él se pueden crear aplicaciones didácticas multimedia dirigidas 0 adaptadas a las necesidades de los alumnos de forma rápida y sencilla. La creación de ejercicios es bastante intuitiva, y permite incluir no sólo textos sino audios y gráficos. El programa permite el diseño y la creación así como la ejecución de las aplicaciones, sin requerir de los usuarios demasiados conocimientos de informática ni grandes equipos para funcionar.

No es un lenguaje de programación, sino un entorno que permite realizar cinco tipos de actividades: puzzles, asociaciones, sopas de letras, crucigramas y actividades de textos. Algunas de estas actividades tienen, a su vez, distintas modalidades: Ias asociaciones pueden ser normales, complejas, de identificación, de exploración, de respuesta escrita, de información; los puzzles o rompecabezas pueden ser de intercambio, dobles, de agujero o de memoria; y entre las actividades de textos las podemos elegir o combinar utilizando las de llenar huecos, completar textos, identificar letras y palabras y ordenar palabras; dando lugar, finalmente, a 19 tipologías básicas. Esta variedad posibilita su utilización desde los tres años de educación infantil hasta el segundo ciclo de enseñanza secundaria.

Los materiales que podemos utilizar pueden ser textos, imágenes, dibujos, sonidos, vídeos digitales, música, etc. Es muy interesante

${ }^{8}$ El proyecto Teleregions es una iniciativa europea de colaboración interregional en aplicaciones telemáticas, en la que participaron Baden Württemberg, Cataluña, Lombardía, Norte de Inglaterra, Ródano Alpes y Upper Austria. 
destacar que, además, se dispone de varias opciones de accesibilidad para personas con necesidades educativas especiales, como son el cursor automático y la posibilidad de utilización del mismo con pulsador, el puntero ampliado y las ventanas de ayuda. Así mismo, resulta oportuno indicar que el programa ofrece el número de aciertos y de errores que ha realizado cada alumno y el porcentaje final de aciertos. Cuenta con un sistema de informes que permite seguir el trabajo individualizado de cada estudiante; los resultados obtenidos pueden integrarse en una base de datos compartida en red, desde donde se pueden obtener perfiles del trabajo de los alumnos.

En otro orden de cosas, señalemos que es un programa en evolución continua, ya que desde el año 1992 aparecen con regularidad versiones mejoradas, nuevas aplicaciones, recursos, etc. En la actualidad hay más de 600 aplicaciones creadas por educadores de distintos países, que superan con creces las 100.000 actividades. En palabras de Francesc Busquets (2000), subrayamos el aspecto cooperativo del entorno:

Desde la primera versión (de 1992) hasta la 3.0 que es la más reciente, ha ido creciendo gracias a la aportación de múltiples ideas y sugerencias de los educadores/as que lo utilizamos para preparar materiales para nuestros alumnos. Pretende ser una herramienta que facilite la creación de materiales multimedia para el aula y la libre difusión e intercambio de las aplicaciones que se vayan desarrollando.

Ese espíritu cooperativo que impregna el proyecto frente a un interés comercial, se refleja también en la licencia Creative Commons, que acompaña todas las actividades de la zona Clic. Dicha licencia significa, como podemos leer en el boletín de noticias $\mathrm{n} . .8$ de la zona $\mathrm{Clic}^{9}$, que se permite el uso y distribución de los materiales pero sí que deberá reconocerse la autoría de las actividades; que está permitido hacer adaptaciones y traducciones de las mismas; que no se permite su comercialización sin permiso de los autores; y que cualquier obra derivada (adaptación o traducción) solamente podrá ser distribuida con una licencia de uso idéntica a la de la obra original.

Como botones de muestra de las aportaciones no españolas, podemos nombrar las dedicadas a la geografía de México y al al fabeto de

\footnotetext{
${ }^{9}$ Disponible en http://clic.xtec.net/llistes/clicnews/clicnews08.htm\#esp.
} 
señas mexicano, a los departamentos de Perú, al vocabulario básico para niños y niñas chinos, a los números en mapudungua (idioma del pueblo mapuche del sur de Chile), al vocabulario básico para niños y niñas rumanos, a las actividades que se llevan a cabo en el departamento de Cerro Largo en Uruguay, y al Pica-pau Amarelo realizado por un colegio de São Paulo en Brasil.

Recientemente ha aparecido la versión J ava del programa Clic 3.0, el denom inadb Jalic. Es un proyecto desoftware libre, que quiere hacer posible su uso en diversas plataformas y sistemas operativos, como, por ejemplo el Linux. De hecho, desde la zona Clic ya es posible descargar los programas para Windows, Linux, Mac y Solaris ${ }^{10}$. El J clic está formado por cuatro aplicaciones:

- Jclic applet. Un «applet» permite incrustar las actividades J clic en una página web.

- J clic player. Es un programa independiente, que, una vez instalado, permite realizar actividades desde el disco duro del ordenador, sin que sea necesario estar conectado.

- J clic autor. Es la herramienta de autor que permite crear, editar y publicar las actividades de una manera más sencilla, visual e intuitiva.

- Jclic reports. Es un módulo de recogida de datos y de generación de informes sobre los resultados de las actividades hechas por los alumnos.

\subsection{LAZONA CLIC}

El espacio web que el Departamento de Educación de la Generalidad de Cataluña dedica a este recurso es La zona Clic ${ }^{11}$, con un interfaz de navegación trilingüe: castellano, catalán e inglés. Las secciones fundamentales que podemos encontrar en la zona Clic son:

- Biblioteca de actividades: se compone de centenares de aplicaciones creadas por profesores de distintos países. Se

\footnotetext{
${ }^{10}$ En http://clic.xtec.net/es/jclic/download. htm.

${ }^{11}$ Tanto al programa como a las aplicaciones y actividades se puede acceder a través de http://clic.xtec.net.
} 
encuentran organizadas tanto por áreas temáticas: ciencias sociales, lenguaje, matemáticas, música y diversos, como por los distintos niveles educativos. Cada actividad dispone de una página explicativa, en la que se pueden observar las características más importantes de la aplicación: un pequeño resumen sobre su contenido, el nivel al que va dirigido, el idioma de instalación, si se dispone de documentación adjunta, la instalación, etc.

- J clic. Se trata de la nueva versión del software Clic, que se apoya en la plataforma J ava en aras de convertirse en un proyecto de software libre.

- Clic 3.0. Es el acceso al programa informático descrito en el apartado anterior.

- Comunidad. Es un espacio para el intercambio de información y para la comunicación entre las personas. Está formada por listas de correo, por enlaces relacionados con el proyecto Clic, por el correo electrónico, por el libro de visitas, etc. Dentro de él, además de enlaces con las comunidades francesa y alemana, encontramos «Argenclic», espacio creado por Fernando Pelillo, con el objetivo de difundir el programa Clic en Argentina, y la página del Clic chileno, que contiene materiales creados por docentes y por especialistas en informática educativa de ese país ${ }^{12}$.

- Documentos. Se encuentran aquí artículos sobre el Clic y el Jclic, manuales técnicos y otros documentos sobre sus aplicaciones.

- Soporte. Es un espacio de ayuda, en el que aparecen las preguntas más frecuentes sobre el proyecto Clic, y sobre el apoyo necesario para la configuración del ordenador.

- Herramientas. Se recopilan aquí instrumentos libres y shareware útiles para la creación, edición o distribución de actividades Clic. Enumeramos los siguientes: el MKInst, como generador de instalaciones; el ClicRT; la versión runtime de Clic 3.0, que sirve para ejecutar los paquetes de

12 Argenclic: http://www.argenclic.com.ar. Clic chileno: http:// www.clicchileno.8m.com. 
actividades; el PaintShop Pro, un editor de gráficos sobradamente conocido; el J asc BatchMaster, que permite modificar conjuntos de archivos gráficos sin tener que abrirlos uno por uno: aumentar o disminuir el número de colores, aplicar filtros y deformaciones, cambiar el formato, etc.; el Audio Compression Manager, para comprin ir archivos WAV; el WordSearch, para generar sopas de letras; el Image Editor, para crear archivos ICO destinados a ser utilizados como iconos en los paquetes de actividades; el editor musical Músic 4.30, muy sencillo, que permite crear archivos MIDI; el AVI Constructor, con el que se pueden crear pequeñas animaciones para incluir en actividades Clic; las Tijeras, que sirven para cortar un archivo grande en trozos pequeños con objeto de copiarlos en disquetes para enviarlos por correo electrónico; y, por último, el CoolEdit'96, editor de sonido digital muy potente, con funciones de grabación, de filtros, de reducción de ruidos, de distorsiones, de ecos, etcétera.

Complementando la zona del Clic, por tradición y con periodicidad anual, se editaba un CD-ROM donde se encontraban todas las aplicaciones aportadas por el profesorado. Este CD-ROM, el último de todos, se denominóSINERA CLIC 2000 , pero debido al incesante aumento de aplicaciones realizadas suprimió su edición y distribución - oficial en todos los centros de Cataluña y gratuita para todos los centros del resto de territorio nacional que lo solicitaran-, por lo que actualmente se descarga desde Internet.

\section{COMO CONCLUSIÓN}

La irrupción de las tecnologías de la información en las escuelas es un fenómeno cada vez más evidente, que aumenta a ritmos elevados y que demanda el acompañamiento de políticas de dotación de equipos y de programas acordes con la nueva realidad. Integrar las TI en los centros supone cambios en sus estructuras organizativas, pero también en la formación del profesorado y en su actitud ante los medios tecnológicos. Se cuenta con un recurso más en los procesos de enseñanzaaprendizaje, y, para aprovechar al máximo sus posibilidades, es necesaria la conjunción de medidas de índole institucional y política, junto a otras de carácter escolar, pero también del esfuerzo y de la formación del profesorado. 
Disponer de aplicaciones que nutran los medios y que se ajusten a los programas establecidos no es tarea fácil, y, además, conseguir que sean los propios profesores los que los preparen, apliquen, comprueben, rectifiquen y ofrezcan a la comunidad educativa el resultado final sin ninguna contrapartida económica, parece una idea imposible de realizar. Sin embargo, hemos mostrado en este artículo que sí es posible; un programa, el $\mathrm{Clic}^{13}$, ha sido capaz de aglutinar a su al rededor desde responsables educativos públicos hasta los propios maestros de nuestras escuelas. Tan plausible iniciativa sobre las TI debería complementarse con aquellas que, realizadas en otros puntos geográficos, desarrollan ciertos aspectos olvidados, marginados o simplemente apartados en el proceso de enseñanza-aprendizaje de nuestros alumnos, como, por ejemplo, las variaciones lingüísticas y culturales, las tecnologías de ayuda a los discapacitados, el acceso de los infomarginados o de los excluidos de las TI, etcétera.

El programa Clic es una aplicación multimedia educativa de origen y de carácter colaborador entre el profesorado, y de distribución gratuita a todo el mundo a través de los distintos servidores que disponen del programa. Martínez y Adell (2004, p. 177) afirman que «una línea de pensamiento que necesita imperativamente incorporar la escuela pública es la subyacente a movimientos como el software libre y de código abierto». Creemos que el caso del Clic se integra en esta línea de pensamiento. Añaden estos autores que «crear materiales, compartirlos por la red, desarrollarlos junto a otros docentes, distribuirlos sin costes [...] son actividades que rompen el círculo vicioso de los libros de texto y las viejas pedagogías». Pues bien, contar con la experiencia del Clic y con otras ofrecidas a través de las plataformas oficiales (CNICE, XTEC, etc.), supone aumentar el bagaje de recursos a disposición del profesorado en aras de romper dicho círculo.

\footnotetext{
${ }^{13}$ Según Ruiz i Tarragó (1999, p. 116), uno de sus mentores, «la coordinación es fundamental para que las inversiones en informática educativa puedan conseguir la finalidad deseada».
} 


\section{BIBLIOGRAFÍA}

BUSQUETS, F. (2000): «Clic: un proyecto cooperativo de producción e intercambio

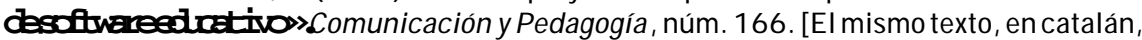
se encuentra en F Busquets (2000): «Clic: un projecte cooperatiu de producció i interc anvi de software educatiu, en WAA: VIII J ornades Didàctiques. Software Educatiu], Vinaròs, Editorial Antinea.

COMES, A. y CATALÀ, A. (2000): «Recursos didácticos en la educación compensatoria. El aula de informática. Aplicaciones del Clic», en WAA: Treballar en equip. 1. a Trobada de Grups de Treball i Seminaris (Torrent). Jornadas de Intercambio de Experiencias en Educación Infantil (Requena), Paterna, Editorial Ecir.

MARTínez, J . y ADELL, J . (2004): «Viejos y nuevos recursos y tecnologías en el sistema educativo», en J . GimenoyJ . Carbonell. (coords.): El sistema educativo. U na mirada crítica, Barcelona, Cisspraxis.

MATESANZ DEL RIO, J. (2000): «Clic: un programa para elaborar aplicaciones educativas», en Educación y medios, 13, pp. 33-36.

QUINTANA, A. (1999): «Programa de Nuevas tecnologías de la Información y la Comunicación (PNTIC)», en WAA: Educación e Internet. Documentos del ler. Congreso Educación e Internet. Educnet '99, pp.121-129, Madrid, Santillana.

RUIZ I TARRAGÓ, F. (1999): «Red telemática Educativa de Cataluña», en WAA: Educación e Internet, pp. 116-120, Madrid, Santillana.

\section{REFERENCIAS WEB (actualizadas a 3-01-05)}

Centro Nacional de Información y Comunicación Educativa:

<http://www.cnice.mecd.es>

Red Telemática Educativa de Cataluña: <http://www.xtec.es>.

Páginas de La zona Clic: <http://clic.xtec.net >.

Recursos yaplicaciones en castellano: <http://members. es.tripod.de/jjmateo>.

Páginas en francés del Clic: <http://www.erasme.org/clic >.

Páginas en alemán del Clic: <http://clic.eduhi.at/>.

Argenclic (Clic en Argentina): <http://www.argenclic.com.ar>.

La página del Clic chileno: <http://www.clicchileno. 8 m.com>.

La página de F. Busquets: < http://www.xtec.es/ fbusquet>. 
J. PEIRATS, C. SALES 